\title{
Prediction of College Students' Psychological Crisis with a Neural Network Optimized by Harmony Search Algorithm
}

\author{
https://doi.org/10.3991/ijet.v17i02.29009
}

Zhiping Jia

Department of Student Affairs, Hebei Chemical \& Pharmaceutical College, Shijiazhuang, China

mjia003efiu.edu

\begin{abstract}
Despite the growing concern with students' mental health education, there are neither extensive research on stressors of psychological crisis, nor targeted research on the psychological crisis warning of college students with different levels of psychological crisis. To solve the problem, this paper aims to explore the psychological crisis warning and physical education (PE) intervention of college students based on artificial neural network (ANN). Firstly, the important evaluation indexes were determined for psychological crisis warning of college students; according to the adverse reactions and performance of college students in physiology, cognition, emotion, and behavior, the index data were processed by partial least squares (PLS) method. Next, a psychological crisis warning model was developed based on the optimized ANN: the harmony search (HS) algorithm was improved based on the differential evolutionary (DE) algorithm, and then used to optimize the backpropagation neural network (BPNN). The proposed model was proved feasible and effective through experiments.
\end{abstract}

Keywords-backpropagation neural network (BPNN), warning of college students' psychological crisis, partial least squares (PLS) method

\section{Introduction}

College students generally have to face the triple pressures of school work, employment, and life; therefore, when their ideality collides with social reality, their psychology is prone to lose balance and generate psychological crises [1-6]. Now, the public has paid more attention to the mental health education of students, and there are more discussions on the mental health education of undergraduates and junior college students [7-13].

College students' psychological crisis develops in three stages: the initial stage, the developing stage, and the severe stage [14-15]. For the different stages, there're certain differences in the adverse reactions and performance of the psychological crisis of college students. Starting from the source of stressors and with the help of effective identification methods, we could discover college students with psychological prob- 
lems as early as possible and give scientific interventions to adjust their mental health status and help them restore balance in their psychology.

Xiong [16] proposed that designing and developing big data analysis prototype systems for college students' mental health education could predict the trend of college students' psychological behavior; and formulating personalized emergency management mechanisms for college students' psychological crisis could effectively improve the real-time performance and effectiveness of the management of college students' psychological crisis. Li and Wang [17] explored the repetitive feature of psychological crisis, and discussed a few aspects including the separation and integration of physical effect, the boundary of strong and weak conditions, the regulation or intermediary mechanisms, and the innovation of experimental paradigms. Huang [18] proposed a psychological crisis warning system based on the C4.5 decision tree algorithm; in the paper, the classification rules generated by the $\mathrm{C} 4.5$ decision tree was fully applied based on quantified mental state of students to facilitate the showing of the relationship between students' mental health indicators and their psychological crisis. Frangos et al. [19] discussed the influence of economic crisis and corruption of the Greek political system on the thinking, lifestyle, and academic progress of modern young Greeks; the negative influence mainly includes the common feeling of abandonment, pessimism, negative beliefs, nihilistic attitudes, and lack of self-confidence, etc. Based on a classification of college students' psychological crises and the nature of crisis events.

In summary, although many field scholars have conducted in-depth research on psychological crisis warning of college students, the research scope of the stressors of psychological crisis is not wide enough, and insufficient consideration has been paid to the different emphases on the psychological crisis warning of college students with different levels of psychological crisis, as a result, the change pattern of college students' psychological crisis with the development of psychological problems couldn't be well investigated. To this end, this paper aims to research the early warning of college students' psychological crisis and PE intervention based on ANN, and the main content of this paper includes the following aspects: 1) Take the adverse reactions and performance of college students in physiology, cognition, emotion, and behavior as important evaluation indexes of psychological crisis warning; 2) Process the data of the evaluation indexes based on the PLS method; 3) Build the psychological crisis warning model based on improved ANN, elaborate on the principles of the improvement and the HS algorithm, and give the execution flow of the BPNN optimized by the improved algorithm. In the last part, this paper used experimental results to verify the effectiveness of the proposed model.

\section{Index determination and data processing}

Combining with the characteristics of college students' psychological crisis and the traits of college students, we can know that college students' psychological crisis has the features of event suddenness, painfulness, helplessness, dangerousness, and dual effect. According to the type of stressors, the psychological crisis of college students 
can be divided into several types: such as the interpersonal relationship type, development prospect type, family and economy type, study and life type, heterosexual relationship type, and physical health type, etc.

As the most important link in the research of the early warning of college students' psychological crisis, the selection of evaluation indexes plays a direct decisive role in the effectiveness of the psychological crisis warning model. Due to the suddenness feature of college students' psychological crisis and the seriousness feature of the consequences, the normal law of study and life of college students can be easily broken, so the adverse reactions and performance of college students in physiology, cognition, emotion, and behavior can be taken as important evaluation indexes to identify whether psychological crisis warning and PE intervention need to be carried out on a college student. In terms of physiology, the main symptoms include decreased body immunity, loss of appetite, insomnia, and sense of suppression in the chest, etc. In terms of cognition, the main symptoms include retardation of thought, inattention, illusions, and memory deterioration, etc. In terms of emotion, the main manifestations include anger, despair, depression, fear, anxiety, indifference, solitary, and depression, etc. In terms of behavior change, the main manifestations include evasion, denial, attack, refusal to help, and use alcohol to relieve sorrow, etc. For the college student psychological crisis warning models of different stages, the emphases of the research on evaluation indexes are different. For students with initial-stage psychological crisis, the research emphasizes on indexes that can reflect recognition and emotions; while for students with severe-stage psychological crisis, the research emphasizes on indexes that can reflect their physiology and behavioral changes.

When constructing regression formula using the PLS method, we only need to select the first $k$ components of $q$ components $o_{1}, o_{2}, \ldots, o_{q}$ to obtain a regression model with an ideal prediction level. The number of required principal components $k$ can be determined by the cross-validity test.

At first, the first $i$ observation points $(i=1,2, \ldots, \mathrm{m})$ of the evaluation indexes of college students' psychological crisis were deleted, and the PLS regression method was adopted to build a model for the remaining $m-k$ observation points. Second, the regression equation fitted after selecting $f$ components was studied, and the $i$-th observation point that had been deleted was substituted into the constructed regression equation to obtain the predicted value of $b_{j}(j=1,2, \ldots, e)$ on the $i$-th observation point. Third, above calculation was repeated for different evaluation index observation points, based on the calculation results, the sum of squared prediction errors of the $j$ th dependent variable $b_{j}(j=1,2, \ldots, e)$ when extracting $f$ components could be calculated using Formula 1 below:

$$
\operatorname{SFE}_{j}(f)=\sum_{i=1}^{m}\left(b_{i j}-\dot{b}_{(i) j}(f)\right)^{2},(j=1,2, \ldots, e)
$$

The sum of squared prediction errors of $B=\left(b_{1}, \ldots, b_{e}\right)^{T}$ can be calculated by Formula 2:

$$
\operatorname{SFE}(f)=\sum_{j=1}^{e} \operatorname{SFE}_{j}(f)
$$


In addition, all the sample points of the score data of evaluation indexes were used to construct a regression equation containing $f$ components. The predicted value of the $i$-th evaluation index score data sample point was recorded as $b_{i j}(f)$, then Formula 3 gives the calculation formula of the sum of squared errors of $y_{i} b_{i}$ :

$$
S Q_{j}(f)=\sum_{i=1}^{m}\left(b_{i j}-\dot{b}_{i j}(f)\right)^{2}
$$

The sum of squared errors of $B$ could be calculated using Formula 4:

$$
S Q(f)=\sum_{j=1}^{m} S Q_{j}(f)
$$

When $S F E(f)$ reaches the minimum value, the number of components needed to be extracted to construct the model at this time is $f$. Under general conditions, $\operatorname{SFE}(f)$ is always greater than $S Q(f)$, and $S Q(f)$ is always less than $S Q(f-1)$, so when extracting the principal components, the smaller the value of $S F E(f) / S Q(f-1)$, the better. Generally, the preset threshold is set to 0.1 , then there is:

$$
\frac{\operatorname{SFE}(f)}{S Q(f-1)} \leq(1-0.1)^{2}=0.9^{2}
$$

The cross-validity can be defined by Formula 6:

$$
W_{f}^{2}=1-\frac{S F E(f)}{S Q(f-1)}
$$

For the observation points of the evaluation indexes of college students' psychological crisis, before the end of each round of calculation, the predicted values of the sample points need to be subject to cross-validity test. If the cross-validity obtained in calculation of the $f$-th iteration is less than 0.081 , it can be considered that the constructed model has reached the accuracy requirement, and the iteration of the algorithm can be terminated. If the cross-validity is greater than 0.081 , it means that the component $o f$ extracted in the $f$-th iteration has a significant marginal contribution, and the calculation of the $f+1$-th iteration is required.

\section{Construction of the psychological crisis warning model based on improved BPNN}

As a meta-heuristic optimization algorithm, the harmony search (HS) algorithm can obtain all solution vectors in the parallel optimization space with the help of intelligent search. This paper adopted HS algorithm and differential evolutionary (DE) algorithm to improve BPNN, which is used to build the algorithm model for quickly and accurately predicting the psychological crisis of college students. 


\subsection{The HS algorithm}

The specific execution steps of the HS algorithm are described in detail as follows:

STEP1: Determine the objective function of the algorithm and initialize relevant parameters. Before execute the algorithm, clarify the status of college students' psychological crisis, and determine the objective function $g(a)$ and its constraints. Assuming $S_{M B}$ represents the size of the harmony memory bank, $M$ represents the scale of decision variables, $\left(a_{K}, a_{V}\right)$ represents the value range of the variables, $T$ represents the number of iterations, $P_{M B}$ represents the retention probability of the harmony memory bank, $P_{T A}$ represents the probability of tone tuning, $T B$ represents the tone bandwidth, the above parameters were initialized.

STEP2: Initialize the harmony memory bank. Figure 1 shows the creation process of the harmony memory bank. Determine $S_{M B}$, the number of solutions in the HS algorithm. The solution vector of the algorithm can be formed by the combination of $M$ solution components. The solution vector $a_{i}(j)=\left\{a_{i}(1), a_{i}(2), \ldots, a_{i}(C)\right\}$ can be generated randomly based on Formula 7:

$$
a_{i}(j)=a^{K}+\left(a^{V}-a^{K}\right) i=1,2, \ldots, S_{M B} j=1,2, \ldots, M
$$

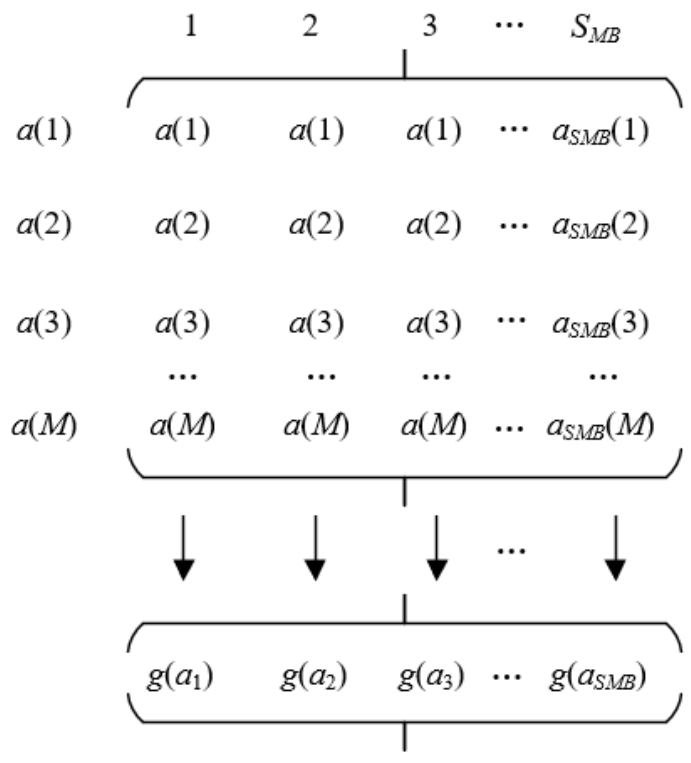

Fig. 1. The creation process of a harmony memory bank

STEP3: Generate new harmony $\mathbf{A}^{*}(\zeta)$. Generate new solution for the HS algorithm based on probability tree. Assuming $C_{1}, C_{2}$, and $C_{3}$ represent three improvisations, create new harmony $A^{*}=\left[a^{*}(1), a^{*}(2), \ldots, a^{*}(C)\right]$ based on $C_{1}, C_{2}$, and $C_{3}$, and calculate the objective function.

Assuming random numbers $s_{1, S_{2}, S_{3}} \in(0,1), s \in\left(1,2, \ldots, S_{M B}\right), \zeta \in(1,2, \ldots, M)$. The operations of $C_{1}$ and $C_{2}$ are shown in Formula 8: 


$$
A^{*}(\zeta)=\left\{\begin{array}{lc}
a_{s}(\zeta) & s_{1} \leq P_{M B} / / C_{1} \\
a^{*}(\zeta) \in\left[a^{K}(\zeta), a^{V}(\zeta)\right] & s_{1}>P_{M B} / / C_{2}
\end{array}\right.
$$

The operation of $\mathrm{C} 3$ is shown in Formula 9:

$$
A^{*}(\zeta)= \begin{cases}a^{*}(\zeta) \pm s_{3} \times T B & s_{2} \leq P_{T A} / / C_{3} \\ a^{*}(\zeta) & s_{2}>P_{T A}\end{cases}
$$

STEP4: Update the harmony memory bank. Figure 2 shows generation process of a new harmony. Compare the worst solution $A^{\prime}$ in the harmony memory bank with the new solution $A^{*}$ generated in the previous step. If $A^{*}$ is better than $A^{\prime}$, then replace $A^{\prime}$ with $A^{*}$; if $A^{*}$ is worse than $A^{\prime}$, then no operation is performed:

$$
\text { if }\left(g\left(A^{*}\right)<g\left(A^{\prime}\right)\right) A^{\prime}=A^{*}
$$

STEP5: Terminate the iterative calculation of the algorithm. If the number of iterations reaches the maximum value $T$ or the convergence conditions have been met, then the iterative operation of the algorithm is terminated; otherwise, return to STEP3 to continue to generate new harmony.

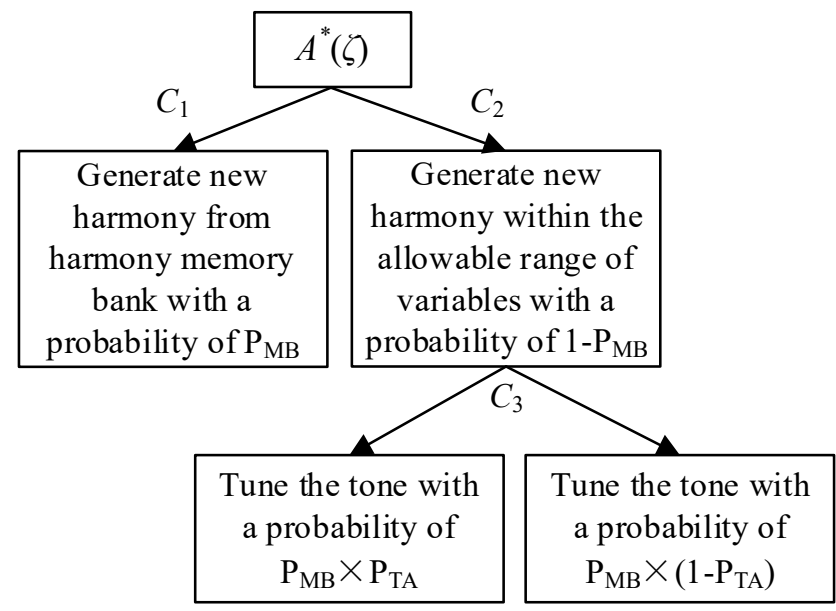

Fig. 2. Generation process of new harmony

\subsection{The DE algorithm}

The DE algorithm solves objective optimization problems through the cooperation and competition between chromosomal individuals in the population. When the population is initialized, Q chromosome individuals can be randomly generated by Formula 11: 


$$
a_{i, l}(0)=k_{l}+\operatorname{rand}\left(v_{l}-k_{l}\right), l=1,2, \ldots, \zeta, i=1,2, \ldots, Q
$$

Assuming: each chromosome individual corresponds to a solution vector, which is represented by $a_{i}=\left[a_{i 1}, a_{i 2}, \ldots, a_{i \zeta}\right]$, wherein $a_{i}$ represents the $i$-th chromosome in the population; $v_{l}$ and $k_{l}$ represent the upper and lower search boundary of the $l$ dimensional variable; $\zeta$ represents the dimension of the problem; rand () is a random number generation function, and the value range of the random numbers generated is $[0,1]$. DE algorithm generally has three steps: mutation operation, crossover operation, and selection operation. Figure 3 shows the flow of the algorithm.

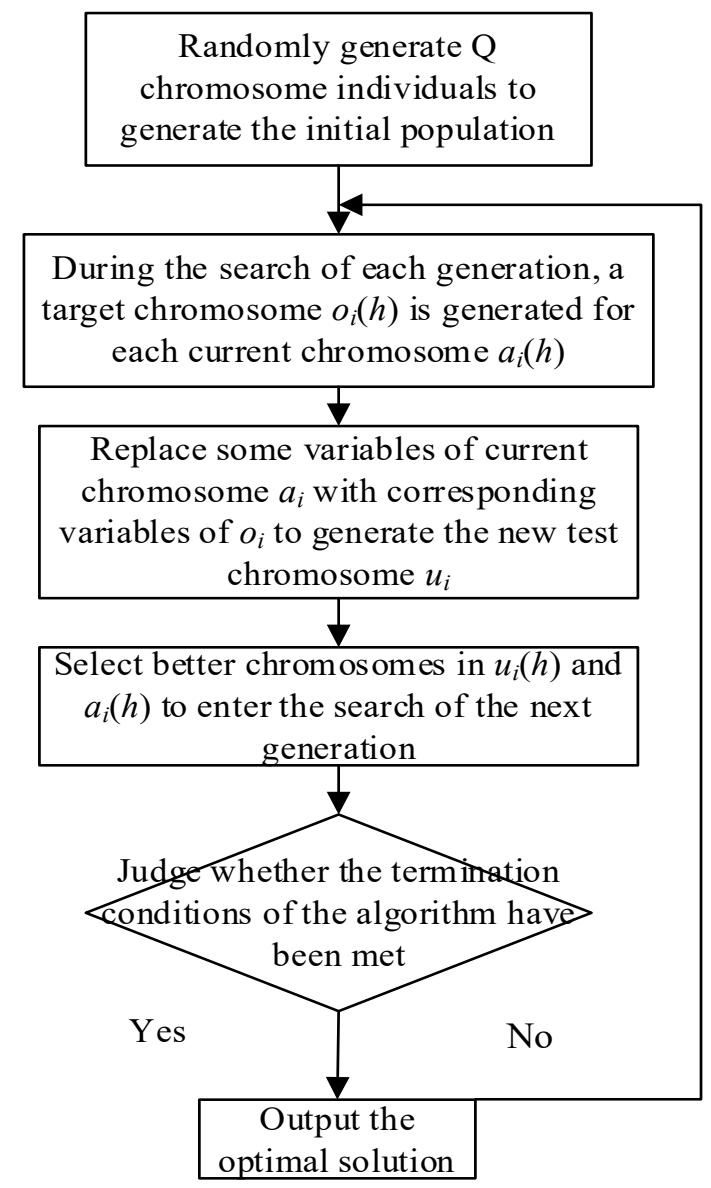

Fig. 3. Execution process of the DE algorithm

Assuming: $h$ represents the number of evolution generations; during the search process of each generation, the mutation operation will generate a target chromosome $o_{i}(h)$ for each current chromosome $a_{i}(h)$. The mutation mechanism of the algorithm could be expressed in the $L X / S M$ form, wherein $L X$ and $S M$ respectively represent the 
type of mutation operation base and the number of difference items during mutation. There're two types of mutation operation base: randomly-selected chromosome or optimal chromosome, which correspond to the two values 1-RS and 2-BEST of $L X$.

Assuming: $s_{1}, s_{2}, s_{3}, s_{4}$, and $s_{5}$ are inequal random integers; $a_{B}(h)$ represents the optimal chromosome in the population of the $h$-th generation; $G$ represents the scaling factor, then Formulas 12-15 give the expressions of common mutation mechanism:

$$
\begin{gathered}
1-R S / 1: o_{i}(h)=a_{s_{1}}(h)+G \cdot\left[a_{s_{2}}(h)-a_{s_{3}}(h)\right] \\
2-B E S T / 1: o_{i}(h)=a_{B_{1}}(h)+G \cdot\left[a_{s_{1}}(h)-a_{s_{2}}(h)\right] \\
1-R S / 2: o_{i}(h)=a_{s_{1}}(h)+G \cdot\left[a_{s_{2}}(h)-a_{s_{3}}(h)\right]+G \cdot\left[a_{s_{4}}(h)-a_{s_{5}}(h)\right] \\
2-B E S T / 2: o_{i}(h)=a_{s_{1}}(h)+G \cdot\left[a_{s_{2}}(h)-a_{s_{3}}(h)\right]+G \cdot\left[a_{s_{4}}(h)-a_{s_{5}}(h)\right]
\end{gathered}
$$

In order to retain better variables in chromosomes while enhancing the local search ability of the algorithm, after the mutation operations are completed, it needs to perform crossover operations, that is, some variables of the current chromosome $a_{i}$ are replaced with the corresponding variables of $o_{i}$ to generate new test chromosome $u_{i}$. This paper adopted two methods of binomial crossover and exponential crossover to perform the crossover operations.

In a binomial crossover operation, at first, for each variable, a random number $s$ within the value range of $[0,1]$ is generated. Assuming $J U$ represents the crossover probability of accepting the variable in $o_{i}(h)$, when $s<J U$, the corresponding component of $o_{i}(h)$ is retained; otherwise, the corresponding component of $a_{i}(h)$ is retained:

$$
\begin{aligned}
v_{i, j}(g) & =\left\{\begin{array}{l}
t_{i, j}(g), r<c r \text { or } j=r n d \\
x_{i, j}(g), \text { others }
\end{array}\right. \\
u_{i j}(h) & =\left\{\begin{array}{l}
o_{i j}(h), s<J U \text { or } j=R \\
a_{i j}(h), \text { others }
\end{array}\right.
\end{aligned}
$$

The $R$ in above formula is used to ensure that at least one-dimensional component is inherited from $o$, and its value range is $[1, \xi]$.

In an exponential crossover operation, at first, select an integer $l$ with value range $[1, \xi]$ as the starting point of crossover, and then determine the number of substitution variables that satisfy Formula 17:

$$
G L\{S J=l\}=(J U)^{l-1} \cdot(1-J U)
$$

In this paper, the selection operations of the algorithm were performed based on the greedy selection method, and better chromosome individuals in $u_{i}(h)$ and $a_{i}(h)$ were chosen to enter the search of the next generation: 


$$
a_{i}(h+1)=\left\{\begin{array}{l}
o_{i}(h), g\left[o_{i}(h)\right]<g\left[a_{i}(h)\right] \\
a_{i}(h), \text { others }
\end{array}\right.
$$

\subsection{Basic theory of ANN}

Figure 4 shows the structure of the constructed three-layer BPNN. As can be seen from the figure, artificial neurons are the basic processing units of the ANN, which has weighting function and characteristics, and can simulate the information processing process of biological neurons. Assuming $a_{i}(i=1,2, \ldots, m)$ represents the input of the $i$-th neuron, $m$ represents the number of input neurons, then Formula 19 gives the input vector of the $j$-th neuron:

$$
A_{j}=\left(a_{1}, a_{2}, \ldots, a_{m}\right)^{T}
$$

Assuming $\omega_{i j}(i=1 、 2 、 \ldots, m)$ represents the connection weight of the input node to the $j$-th neuron in the hidden layer, Formula 20 gives its expression:

$$
\omega_{j}=\left(\omega_{1 j}, \omega_{2 j}, \ldots, \omega_{m j}\right)^{T}
$$

Assuming the threshold of the $\mathrm{j}$-th neuron is $\gamma \mathrm{j}$, then, its input weighted sum, namely the input excitation of artificial neurons pj is given by Formula 21:

$$
p_{j}=\sum_{i=1}^{m} a_{i} \omega_{i j}-\gamma_{j}
$$

Assuming $\Gamma(\cdot)$ represents the transfer function used to describe the nonlinear relationship between the input and output of neurons, then Formula 22 gives the expression of the output of the $j$-th neuron:

$$
b_{j}=\Gamma\left(p_{j}\right)
$$

According to above formula, after $p_{j}$ is processed by the transfer function, the final output value $b_{i}$ of the neural network can be obtained. 


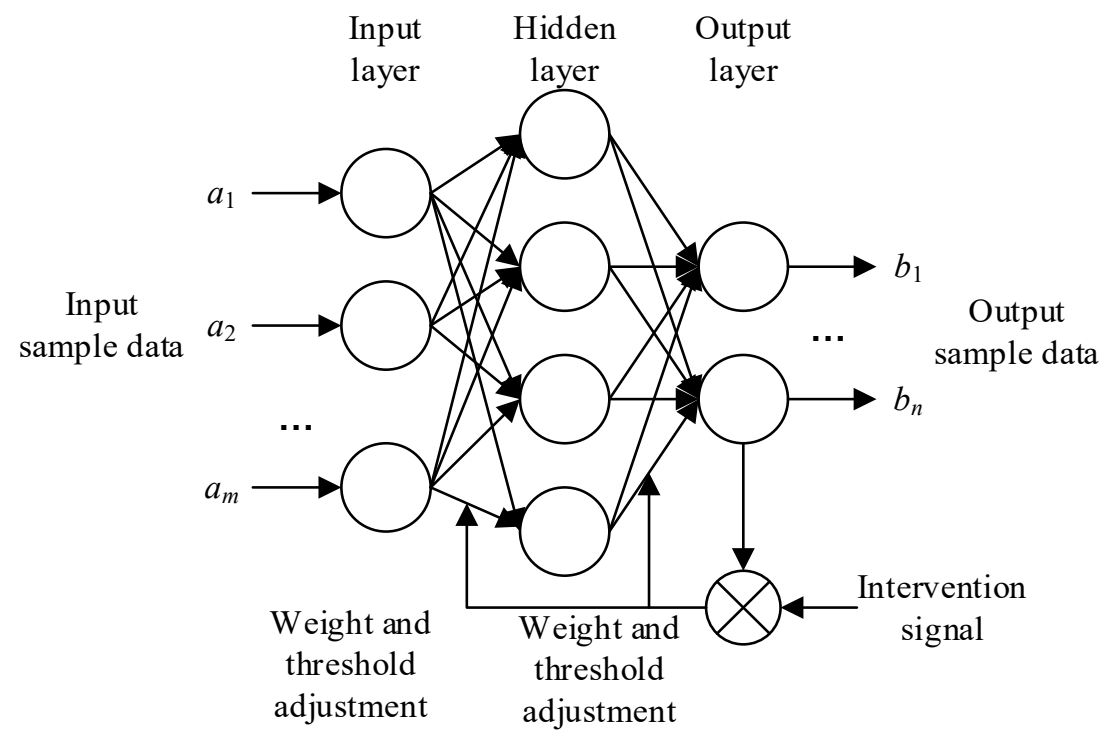

Fig. 4. Structure of the constructed three-layer BPNN

\subsection{The Improved BPNN}

This paper improved the traditional HS algorithm based on the mutation mechanism of the DE algorithm. That is, in the original improvisation $C^{3}$ tuning stage, a new tone $C^{3 *}$ was generated through two pairs of differential mutation operations of the mutation mechanism of $\mathrm{DE}$ algorithm, and $C^{3}$ was replaced by $C^{3 *}$. Assuming $N D$ represents normal distribution, the formation of $C^{3^{*}}$ was divided into two steps, at first, the differential mutation vector $A_{D M}$ shown as Formula 23 was generated:

$$
A_{D M}(\mu)=N D(0.45,0.35) \times\left[\left(a_{s 1}-a_{s 2}\right)+\left(a_{s 3}-a_{s 4}\right)\right]
$$

$$
S_{1}, S_{2}, S_{3}, S_{4} \in\left(1, S_{M B}\right)
$$

where, $A_{D M}$ contains two differential mutation pairs. Then, the tone was tuned referring to the new probability of tone tuning $P_{T A}{ }^{*}$, as shown in the following formula:

$$
\begin{aligned}
& A^{*}(\mu)=\left\{\begin{array}{lc}
a^{*}(\mu)+a_{D M}(\mu) & s_{2} \leq P_{T A}{ }^{*} \\
a^{*}(\mu) & s_{2}>P_{T A}{ }^{*}
\end{array}\right. \\
& P_{T A}{ }^{*} \in\{0,0.1,0.2,0.3,0.4,0.5,0.6,0.7,0.8,0.9,1\}
\end{aligned}
$$

In order to verify the superiority of the improved algorithm, this paper tested its global complexity in fixed dimensions. Formulas 26-30 give five commonly-used test functions: 


$$
\begin{gathered}
\Omega_{1}(a)=\sum_{i=1}^{m-1}\left[100\left(a_{i+1}-a_{i}^{2}\right)^{2}+\left(a_{i}-1\right)^{2}\right] \\
\Omega_{2}(a)=\sum_{i=1}^{m}\left[a_{i}^{2}-10 \cos \left(2 \pi a_{i}\right)+10\right] \\
\Omega_{3}(a)=2.5 \times 10^{-3}\left[\sum_{i=1}^{m} a_{i}^{2}-\prod_{i=1}^{m} \cos \left(\frac{a_{i}}{\sqrt{i}}\right)+1\right] \\
\Omega_{4}(a)=-20 e^{-\frac{\sqrt{\frac{1}{m}} \sum_{i=1}^{m} a_{i}^{2}}{5}}-e^{-\frac{1}{m_{i=1}^{m}} \cos 2 \pi a_{i}}+20+r \\
\Omega_{5}(a)=-\sum_{i=1}^{m}\left(a \sin \sqrt{\left|a_{i}\right|}\right)
\end{gathered}
$$

The initial position of the learning mode of BPNN determines its convergence. In order to effectively avoid local minima during the convergence process, the initial connection weight of the neural network needs to be adjusted appropriately. This paper used the method of optimizing connection weight and threshold to replace the original random initialization of network parameters. Assuming $\Phi_{i}^{l}$ represents the deviation between the actual output of the network and the expected output of the $l$-th learning mode; $S D^{l}$ represents the mean square error of $\Phi_{i}^{l}$, then Formula 31 gives the derivation formula of network error:

$$
\begin{aligned}
& S D^{l}=\frac{1}{2} \sum_{o=1}^{w}\left(\Phi_{o}^{l}\right)^{2} \\
& =\frac{1}{2} \sum_{o=1}^{w}\left(b_{o}^{k}-d_{o}^{c}\right)^{2} \\
& =\frac{1}{2} \sum_{o=1}^{w}\left\{b_{o}^{l}-g\left[\sum_{j=1}^{t} u_{j o}\left(\sum_{i=1}^{t} u_{i j} a_{i}^{l}-\gamma_{j}\right)-\phi_{o}\right]\right\}^{2} \\
& =\frac{1}{2} \sum_{o=1}^{w}\left\{b_{o}^{l}-g\left[\sum_{j=1}^{t} u_{j o}\left(\sum_{i=1}^{m} q_{i j} a_{i}^{l}-\gamma_{j}\right)-\phi_{o}\right]\right\}^{2}
\end{aligned}
$$

This paper adopted the improved HS algorithm to optimize the connection weight of the BPNN, that is, the network training process was regarded as the process of searching for the optimal solution in the weight value space.

Assuming: $E S$ represents the sum of squared errors between the expected network output and the actual output; the set of connection weights and thresholds of the BPNN could be equivalent to each harmony vector in the harmony memory bank. At the same time, the objective function of the BPNN optimized by the improved HS algorithm, namely the fitness value, was determined by Formula 32:

$$
E S=\sum_{z=1}^{Z} \sum_{i=1}^{P}\left(b_{i}^{z}-d_{i}^{z}\right)^{2}
$$


Paper-Prediction of College Students' Psychological Crisis with a Neural Network Optimized by...

Smaller $E S$ value indicates better chromosomes, on the contrary, greater $E S$ value indicates worse chromosomes.

\section{$4 \quad$ Experimental results and analysis}

According to the adverse reactions and performance of college students in physiology, cognition, emotion, and behavior, 23 evaluation indexes were selected for the early warning of psychological crisis; then, based on the principle of PLS, corresponding programs were written in MATLAB to extract the components of the 23 evaluation indexes. Table 1 summarizes the regression coefficients of evaluation indexes to the components.

The explanatory ability and cumulative explanatory ability of PLS components to dependent variables could be obtained through calculation, in this paper, the explanatory ability of the 2 components extracted by PLS was 0.8242 and 0.1346 respectively, and the cumulative explanatory ability was 0.9588 , that is, the extracted components can explain $95.88 \%$ of the dependent variables, so the operation of the optimal PLS components was scientific and reasonable.

In order to verify the superiority of the improved algorithm, this paper designed several reference models (Reference model 1: optimized by traditional HS algorithm; Reference model 2: optimized by improved HS algorithm; Reference model 3: optimized by the hybrid algorithm of traditional HS algorithm and DE algorithm) to conduct comparative experiments on the reference models and the proposed model. During the experiment, for the prediction of each problem of college student's psychological crisis, the algorithms before and after optimization were executed for 30 times each, and each time, the initialized parameter values were the same. Meanwhile, for the above 5 test functions, the maximum calculation times $N_{\max -c}$ and the minimum error function value $V_{\text {min-e }}$ were set as two algorithm termination conditions, based on $N_{\max -c}$ and $V_{\text {min-e }}$, the optimization performance of the algorithms could be evaluated.

Figure 5 gives the test results of different algorithms, showing the distribution of experience cumulative functions of test functions in case of fixed dimensions with respect to different algorithms, and the accuracy of the number of successes in predicting the psychological crisis of college students was $10^{-8}$. According to the figure, after all models went through 8000-20000 iterations, the proposed model outperformed the other three algorithm models.

This paper used the $\operatorname{train}()$ function to train the constructed neural network model. Before training, the maximum training times and learning rate of the network were set to 1000 and 0.01 respectively, the target threshold of output errors was set as $10^{-6}$, and other parameters took default values. Figure 6 shows the error curves of the training process of the constructed neural network. According to the figure, the constructed neural network exhibited good convergence performance and can meet the requirements for the early warning of college students' psychological crisis.

This paper used the $\operatorname{sim}()$ function to simulate the trained neural network, then, the simulation results after subject to denormalization operation were compared with the 
original predicted data, and the comparison results are given in Figure 7. According to the figure, the simulation results were very close to the original predicted data, indicating that the trained neural network achieved an ideal effect on the prediction of college students' mental health status.

Table 1. Regression coefficients of evaluation indexes to the components

\begin{tabular}{|l|c|c|l|c|c|}
\hline & Q1 & Q2 & & Q1 & Q2 \\
\hline$E W_{1}$ & -0.3242 & -0.0532 & $E W_{13}$ & -0.1427 & -0.2753 \\
\hline$E W_{2}$ & -0.2765 & 0.1785 & $E W_{14}$ & -0.1592 & -0.3785 \\
\hline$E_{3}$ & -0.1957 & 0.2736 & $E W_{15}$ & -0.2351 & -0.2743 \\
\hline$E_{4}$ & -0.3542 & -0.0175 & $E W_{16}$ & -0.1254 & 0.2785 \\
\hline$E W_{5}$ & -0.3756 & -0.0752 & $E W_{17}$ & -0.2362 & -0.075 \\
\hline$E W_{6}$ & -0.3278 & -0.1752 & $E W_{18}$ & -0.2792 & -0.1233 \\
\hline$E_{7}$ & -0.2351 & -0.1936 & $E W_{19}$ & -0.1242 & 0.2753 \\
\hline$E W_{8}$ & -0.1257 & -0.1751 & $E W_{20}$ & -0.0875 & -0.1052 \\
\hline$E_{9}$ & -0.0575 & -0.5752 & $E W_{21}$ & -0.1372 & -0.1239 \\
\hline$E W_{10}$ & -0.2375 & 0.0769 & $E W_{22}$ & 0.0354 & -0.2672 \\
\hline$E W_{11}$ & -0.2239 & -0.1753 & $E W_{23}$ & 0.0121 & -0.1142 \\
\hline$E W_{12}$ & -0.1678 & 0.1342 & & & \\
\hline
\end{tabular}

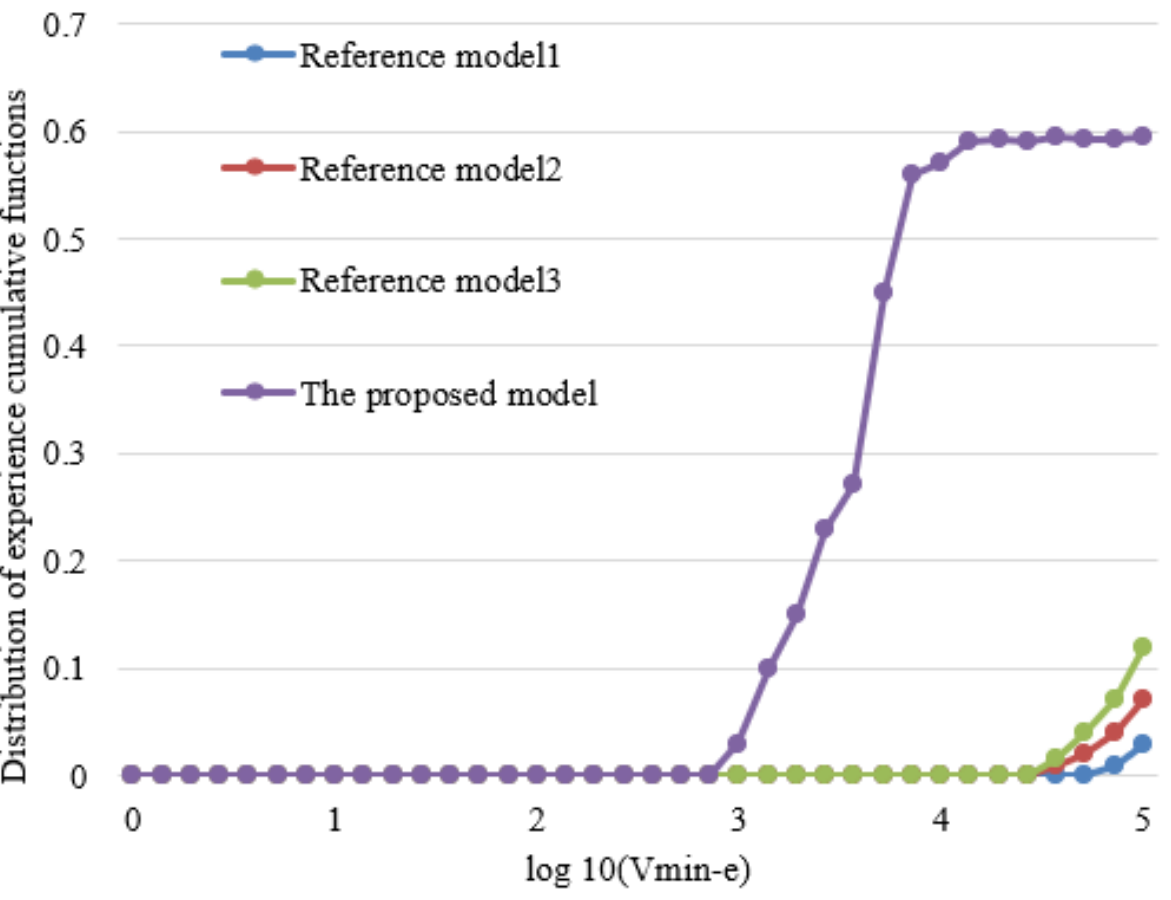

Fig. 5. Test results of different algorithms 
Paper-Prediction of College Students' Psychological Crisis with a Neural Network Optimized by...

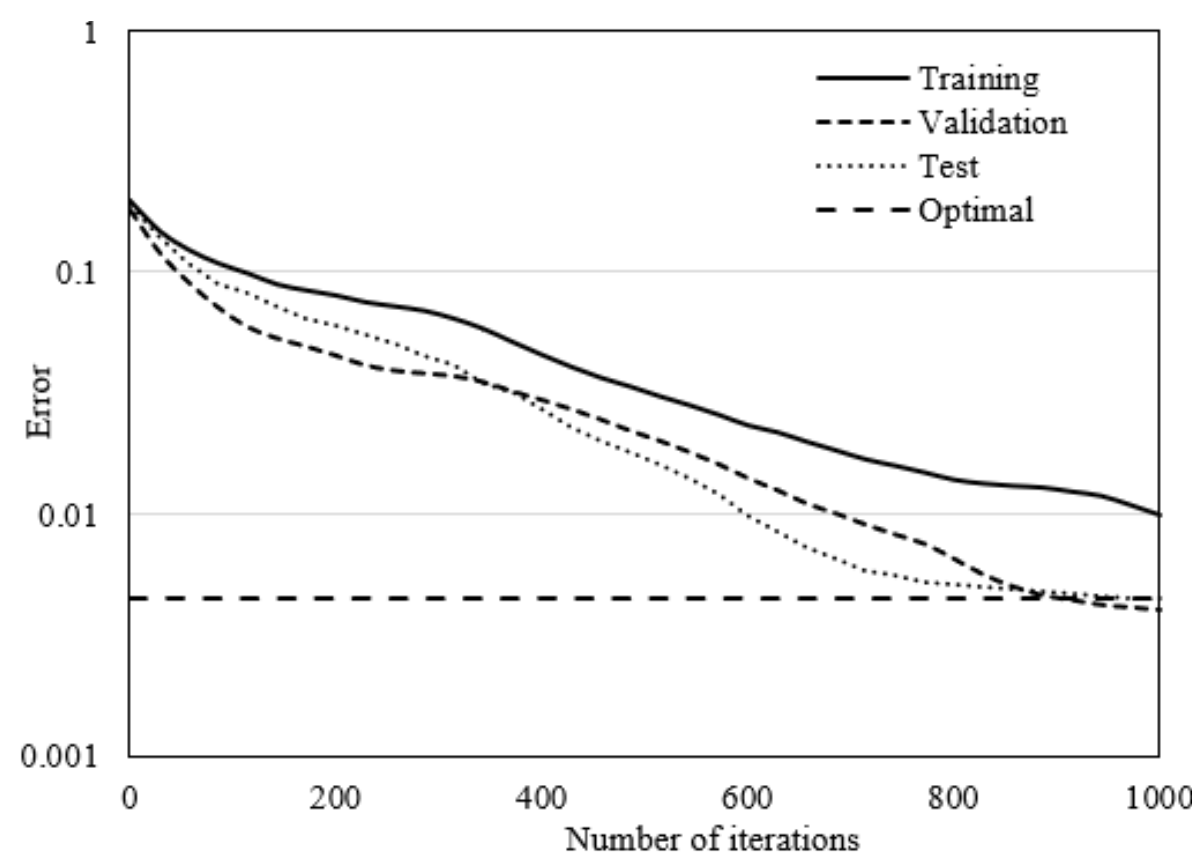

Fig. 6. Error curves of training process of the constructed neural network

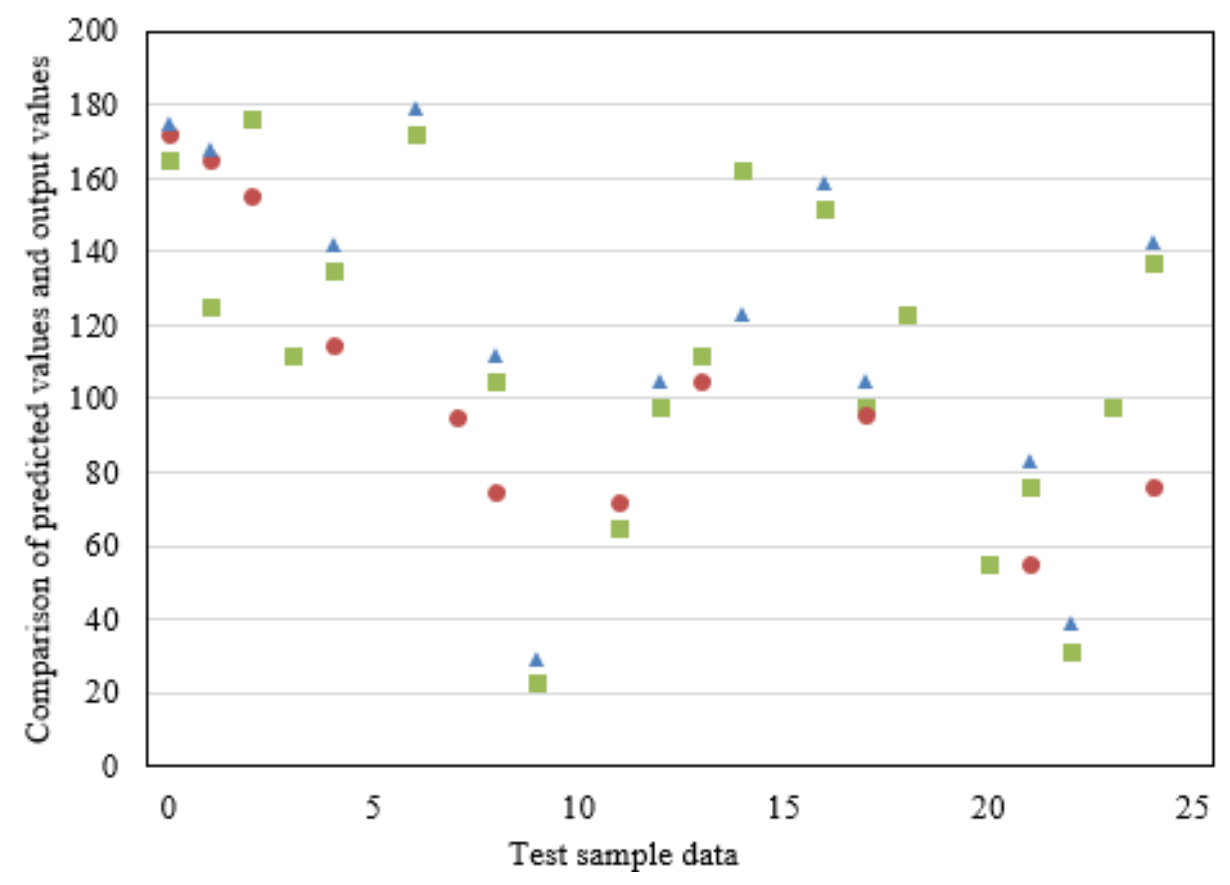

Fig. 7. Comparison of predicted values and actual output values of the neural network after training 


\section{Conclusion}

This paper researched the psychological crisis warning and PE intervention of college students based on ANN. At first, this paper sorted out the adverse reactions and performances of college students with psychological problems in physiology, cognition, emotion, and behavior; then, based on the results sorted out, important evaluation indexes were determined, and the PLS method was adopted to process the data of the determined evaluation indexes. After that, an ANN model was built for the early warning of college students' psychological crisis, the ANN was optimized by an improved HS algorithm, and the execution flow of the BPNN optimized by the improved HS algorithm was given in the text. The experimental results gave a summary of the regression coefficients of evaluation indexes to the components. Through calculation, the explanatory ability and cumulative explanatory ability of PLS components to the dependent variables could be obtained, which had verified that the operations of the optimal PLS components were scientific and reasonable. Moreover, this paper also gave the distribution of experience cumulative functions of test functions in case of fixed dimensions with respect to different algorithms, which had proved the superiority of the proposed model. At last, the error curves of the training process of the neural network, the comparison results of predicted values and actual output values, and the linear regression processing results all demonstrated the effectiveness and feasibility of the constructed model.

\section{$6 \quad$ References}

[1] Liu, J., Shi, G., Zhou, J., Yao, Q. (2021). Prediction of college students' psychological crisis based on data mining. Mobile Information Systems. https://doi.org/10.1155/2021/ 9979770

[2] Antelo, I.F., Gordillo, I.C., Parra, G.M. (2020). Determining Factors in the Perception of Cyberbullying in Victimized Adolescents: Psychoeducational Implications, International Journal of Emerging Technologies in Learning, 15(24): 38-51.

[3] Zhang, Y. (2020). Research on college students' psychological crisis early warning method based on fuzzy clustering algorithm. In 2020 International Conference on Computers, Information Processing and Advanced Education (CIPAE), 26-30. https://doi.org/10.1109/ CIPAE51077.2020.00015

[4] Shen, C.C., Qi, A.L. (2020). An Adaptive Learning Mode of "Public Psychology" Based on Creative Thinking with Virtual Simulation Technology, International Journal of Emerging Technologies in Learning, 15(23): 131-144. https://doi.org/10.3991/ijet.v15i23.18957

[5] Chang, Q., Xinyi, L. (2017). Psychological crisis of college students in a computer network environment. AGRO Food Industry Hi-Tech, 28(1): 1963-1966.

[6] Luo, Y., Sun, Y., Chen, X., Ren, C., Yao, X. (2018). School emergencies and college student psychological crisis intervention. In International Conference on Man-MachineEnvironment System Engineering, 41-47. https://doi.org/10.1007/978-981-13-2481-9 6

[7] Sockalingam, S., Kirvan, A., Pereira, C., Rajaratnam, T., Elzein, Y., Serhal, E., Crawford, A. (2021). The role of tele-education in advancing mental health quality of care: A content analysis of project ECHO Recommendations. Telemedicine and E-Health, 27(8): 939-946. https://doi.org/10.1089/tmj.2021.0122 
Paper-Prediction of College Students' Psychological Crisis with a Neural Network Optimized by...

[8] Da Silva, C., Torres, A., Kapralos, B., Peisachovich, E., Dubrowski, A., Baltazar, V., Caraballo, N. (2021). Person-centered virtual serious games: Mental Health Education. In 2021 IEEE 45th Annual Computers, Software, and Applications Conference (COMPSAC), 1383-1384. https://doi.org/10.1109/COMPSAC51774.2021.00196

[9] Gong, K. (2020). Extensible strategies and their performance for mental health education in colleges. International Journal of Emerging Technologies in Learning, 15(9): 205-218. https://doi.org/10.3991/ijet.v15i09.14037

[10] Saparkyzy, Z., Maygeldiyeva, S., Maygeldiyeva, Z., Taiman, S., Daribaeva, S. (2020). Influence of innovative health-saving education technologies on improvement of mental activity of students. In E3S Web of Conferences, 159: 09011. https://doi.org/10.1051/ e3sconf/202015909011

[11] Mantzios, M., Cook, A., Egan, H. (2019). Mental health first aid embedment within undergraduate psychology curriculums: an opportunity of applied experience for psychology students and for enhancing mental health care in higher education institutions. Higher Education Pedagogies, 4(1): 307-310. https://doi.org/10.1080/23752696.2019.1640631

[12] Djimantoro, M.I. (2018). Multisensory experience for mental health in higher education classroom design. In IOP Conference Series: Earth and Environmental Science, 195(1): 012081. https://doi.org/10.1088/1755-1315/195/1/012081

[13] Evans, T.M., Bira, L., Gastelum, J.B., Weiss, L.T., Vanderford, N.L. (2018). Evidence for a mental health crisis in graduate education. Nature biotechnology, 36(3): 282-284. https://doi.org/10.1038/nbt.4089

[14] Sebastian, J., Richards, D. (2017). Changing stigmatizing attitudes to mental health via education and contact with embodied conversational agents. Computers in Human Behavior, 73: 479-488. https://doi.org/10.1016/j.chb.2017.03.071

[15] Boer, M., Stevens, G.W., Finkenauer, C., de Looze, M.E., van den Eijnden, R.J. (2021). Social media use intensity, social media use problems, and mental health among adolescents: Investigating directionality and mediating processes. Computers in Human Behavior, 116: 106645. https://doi.org/10.1016/j.chb.2020.106645

[16] Xiong, W. (2020). Identification and early warning of college students' psychological crisis based on big data. In Data Processing Techniques and Applications for Cyber-Physical Systems (DPTA 2019), 23-28. https://doi.org/10.1007/978-981-15-1468-5 4

[17] Li, Y., Wang, Y. (2019). Evaluation and analysis of two sources of psychological repeatability crisis based on machine vision. In 2019 International Conference on Machine Learning, Big Data and Business Intelligence (MLBDBI), 159-165. https://doi.org/10.1109/ mlbdbi48998.2019.00037

[18] Huang, Z. (2015). A kind of psychological crisis warning system based on C4.5 decision tree algorithm. Engineering Technology, Engineering Education and Engineering Management - International Conference on Engineering Technology, 2014: 1013-1015. https://doi.org/10.1201/b18566-233

[19] Frangos, C., Sotiropoulos, I., Orfanos, V., Toudas, K., Gkika, E. (2012). The effects of the Greek economic crisis on eating habits and psychological attitudes of young people: A sample survey among Greek university students. In Proceedings of the World Congress of Engineering, 1: 4-6.

\section{$7 \quad$ Author}

Zhiping Jia is currently a lecturer in Hebei Chemical \& Pharmaceutical College. She graduated from Hebei Normal University with a master's degree in psychology, 
Paper_-Prediction of College Students' Psychological Crisis with a Neural Network Optimized by...

research direction including basic psychology, education and development psychology, counseling psychology, has published a core paper in psychology, one SCI, more than 20 other provincial papers, psychological professional related utility model patents 4,1 national invention patent.

Article submitted 2021-10-18. Resubmitted 2021-12-15. Final acceptance 2021-12-17. Final version published as submitted by the author. 